\title{
Infectious diseases as complications following spinal cord injury
}

\section{John Nolan'}

Tjokorda Gde Bagus Mahadewa

'Faculty of Medicine, Udayana University, Bali, Indonesia

2Department of Neurosurgery,

Faculty of Medicine, Udayana University, Sanglah General Hospital, Bali, Indonesia
Cite this article:

Nolan J, Mahadewa TGB. Infectious diseases as complications following spinal cord injury.

Neurologico Spinale Medico Chirurgico.

202I.4(I): I-6

DOI: I0.36444/nsmc.v4il.I22

Corresponding author:

John Nolan

Faculty of Medicine,

Udayana University, Bali, Indonesia

Jalan Kerta Dalem Prestige Nomor 2,

Denpasar, Indonesia

johnnolan@student.unud.ac.id

\begin{abstract}
Background. Infectious complications in spinal cord injury (SCI) patients can increase the mortality and morbidity of the patients. The inability to do daily activity is the main cause of the occurrence of infectious complications. Good understanding and high awareness are needed to notice some infectious complications which are difficult to be diagnosed.
\end{abstract}

Methods. This paper is a literature review which was done by reviewing and searching journals with "infectious complication”, "spinal cord injury", "infection” on the search engines. The authors found 52 articles are suitable to be composed as references for this paper.

Results. Several infectious complications may occur following the incidence of SCI and have a lot of disadvantages. The causes of these infections are multifactorial. Urinary tract infection, infected pressure ulcer, pneumonia, and some other infectious diseases may appear as complications. Extra care in hygiene and sanitation is needed, besides, high awareness is needed to discover these complications.

Conclusion. Monitoring post-SCI complications are complex and difficult. A high level of suspicion should be raised with frequent aseptic actions and procedures. Appropriate management including non-surgical or surgical procedures can be chosen to achieve the best outcomes. Early diagnosis and management are critical for the best treatment results.

Keywords: Infectious complication, Spinal cord injury, Infection

\section{Introduction}

Spinal cord injury (SCI) is a complex and life-disrupting medical condition that was mostly associated with multiple traumas. ${ }^{1,2}$ There are distinctive data about the incidence of SCI all around the world, however, the study reported that 12.000 new cases are noted annually. ${ }^{3,4}$ SCI could be happened due to injuries of any level of the spinal cord resulting in irreversible or reversible casualties. ${ }^{4}$ The functional outcomes of SCI patients usually depend on the completeness disruption level of the spinal cord. ${ }^{5}$ Patients with SCI usually need some more time to stay in the hospital following their needs of treatment and rehabilitation. This prolonged stay in the hospital may lead to an increased risk of developing infectious complications. ${ }^{6,7}$ Not only do infectious complications hold a bigger risk of morbidity and mortality but also precipitate higher cost of management. ${ }^{8}$ 
SCI patients may acquire complications through several factors. Constant usage of invasive medical devices including urinary and intravascular catheters may cause community-acquired infections. ${ }^{6,9}$ Besides, incompetence to cough, changing position, walk, sit, and some other risk factors may contribute to predisposition in a different type of infectious complications. A high morbidity burden is produced by this complication apart from death. The most common infectious complications are systemic infections including respiratory tract infection, urinary tract infection, bloodstream infection, etc. ${ }^{10}$ The inability to move, sit, and change position may cause pressure ulcers and skin infections, osteomyelitis may also occur. ${ }^{11}$ Other infection, such as pneumonia, often occurs to many individuals with SCI need intubation with a mechanical ventilator. The usage of a prolonged mechanical ventilator in the intensive care unit may provoke the occurrence of ventilator-associated pneumonia. ${ }^{12,13}$

Antibiotic therapy is should also be concerned as many cases nowadays with antimicrobial drug resistance cases. Thus, the management of the post-SCI patient is challenging. ${ }^{14}$ Adequate antimicrobial therapy is important as it may control the ongoing infection. ${ }^{15}$ Few drug-resistant microorganisms, such as methicillinresistant Staphylococcus aureus (MRSA), Pseudomonas aeruginosa, Acinetobacter, and extended-spectrum $\beta$-lactamase (ESBL)-producing Enterobacteriaceae especially in developing countries are needed to treat with appropriate antimicrobial choice for the reason of overcoming dreadful adverse outcomes. Initial antibiotic treatment for the first 24 hours is crucial. ${ }^{14,15}$

Many complications can happen following the SCI. One of the most important and often happens is infection. Infection complications themselves are varied. Multiple system organs could be affected by this complication since many factors may play a role in the process. Clinicians need to know this complication following individuals with SCI history. It is also essential for clinicians to have the ability to calculate and predict infection being one cause of increased morbidity and mortality. Good understanding and brief awareness about the complications are needed. Hence, this paper is made to explain the infectious complications in post-SCI individuals to gain more insight into these problems.

\section{Mechanism of Infection in post-SCI individuals}

Multiple organs might be affected following the injury. The occurrence of complications can be short and long-term throughout the whole body. Multifactorial aspects may contribute to infectious complications. ${ }^{16}$ There are many pro-inflammatory mediators released in the local inflammatory microenvironment following the accumulation of degenerated neurons, myelin sheath, damaged endothelial cells, stimulated glial, and infiltrating cells. This intraspinal inflammation can trigger a life-threatening situation called systemic inflammatory response syndrome (SIRS). ${ }^{17-19}$ The whole process of post-injury can cause another emanation in the immune interplay with the spinal cord. ${ }^{20}$ SCI-induced immune depression syndrome (SCI-IDS) is a major factor causing the complication, this phenomenon happens because of the sympathetic nervous system dysregulation and immune organ flawed. ${ }^{21}$ The disruption of supraspinal control over the hypothalamic-pituitary-adrenal axis is occurring in thoracolumbar spinal cord projections to sympathetic ganglion because of SCI. The progress is resulting in sympathetic nervous system dysfunction. ${ }^{22,23}$ Not only does SCI-IDS worsen the neurological function but also diminish the circulating immune response including both innate and adaptive immune response in rats with SCI. ${ }^{24}$ There was a pilot study conducted with 16 SCI patients that showed a lower level of monocytes, $\mathrm{T}$ lymphocytes, and B lymphocytes without decreasing the level of granulocytes in 24 hours post-injury. ${ }^{25}$

Several organs related to infection might be affected such as the lung, spleen, urinary system. ${ }^{26}$ The exact theory behind this immunedepression is still unknown, however, there is a theory that the injury causes interplay trouble between the immune system and CNS. A great number of inflammation mediators in several activations including the hypothalamus-pituitary-adrenal (HPA) axis and the sympathetic nervous system (SNS). The process in the HPA axis may include glucocorticoids resulting in a natural killer cell (NK cell) or antigenpresenting cell (APC), these mechanisms may cause impairment in antimicrobial defense as APC induces the release of an inflammatory mediator. SNS pathway induction may be causing the great amount of catecholamines release as it may induce type 1 T-helper cells and NK cells resulting in the interferon- $\gamma$ and apoptosis activity. These are causing impairment in anti-inflammatory response through the abundant amounts of glucocorticoids and catecholamines release. ${ }^{22,23,27}$ Anti-inflammatory impairment is resulting in an increased risk of infection as the function of antimicrobial defense is impaired. Loss of neurological function also takes part since the loss of protective reflexes precipitating dysphagia, aspiration, bladder dysfunction, and invasive medical procedures enhance the bigger risk of infection. ${ }^{27}$ There is another hypothesis describing the potential of efferent vagus nerve fibers, provoking the delivery of acetylcholine. Also, it may inhibit the activity of macrophage by competitively attaching to specific nicotinic acetylcholine receptors. ${ }^{27}$ 


\section{Infectious complications following SCI}

\section{Urinary tract infection (UTI)}

Impaired supraspinal control in the urinary system may affect the bladder and result in a neurogenic bladder. The condition may lead to in patient's inability to emptying the bladder. ${ }^{28,29}$ Paraplegia due to SCI also contributes to the disease. Multifactorial reasons are accountable, such as a curtailed sensation of classical UTI symptoms, incomplete bladder emptying, frequent catheterizations, or chronic urinary tract catheters. ${ }^{30}$ Becoming the second leading cause of death in SCI individuals, clinicians should be aware of this complication. UTI cause serious complication following the UTI progression to sepsis and septic shock if left undiagnosed and untreated. ${ }^{28,29,31}$

Due to the difficulty in detecting early symptoms, all relevant conditions should be evaluated before a fever develops in UTI. ${ }^{32}$ The longer usage of a foley catheter should also be a concern in developing UTI. Biofilm of bacteria may cause cystitis and $80 \%$ of UTIs are because of the catheter application. Compared to short-term catheterization, the long-term catheter has a higher risk of infection, additionally nitrofurazone-coated and the silver alloy-coated catheter can reduce the risk of infection in the short-term usage of a catheter. ${ }^{33}$ UTI in individuals with SCI are also followed by several risk factors, such as reflux of vesicoureteral, postvoiding residuals, outlet obstruction, urinary tract stones, and bladder overdistension. ${ }^{34}$

Colonization of resistant organisms because of prolonged hospital stay held a new challenge as the treatment of these UTI patients may be difficult. Hence, making sure the catheter in a closed condition is crucial so that no organisms can enter the system. The duration of the catheter is also critical, periodic catheter and suprapubic catheter could be used as a replacement for an indwelling catheter to reduce the risk of infection. ${ }^{35}$ Early recognition and extra attention should be taken by the clinician in case of this infectious complication. Evaluating the hygiene of the urinary tract is necessary including the evaluation of functional and structural disorders. Antibiotic prophylaxis is recommended to be given immediately before applying any invasive medical devices. ${ }^{36}$

\section{Pressure ulcer}

An infected pressure ulcer is a frequent infectious complication in SCI individuals. ${ }^{11}$ This complication is serious and important as it cause long-term morbidity and can affect quality of life, even causing mortality. ${ }^{37}$ This chronic lesion attenuates SCI patients and prolongs the hospital's course. The prevalence among hospitals regarding infected pressure ulcers varies from 25\% to 66\%. ${ }^{38}$ Multiple organisms can be found in the ulcer, including aerobic and anaerobic microorganisms. The condition of necrotic tissue with the addition of a certain smell has been defined as anaerobic isolation in the deep tissue. The most common predilection sites are ischial tuberosity, the trochanter, and the sacrum which commonly form on the bony prominence. ${ }^{11,39}$ There are many risk factors in the contribution of pressure ulcers such as cervical collar and spinal board causing confined activity, paralysis due to complete cord injury, history of smoking, diabetes mellitus, hypoalbuminemia, nursing home residence, or prolonged hospital stay. Besides, sensation loss, wet areas due to fecal or urinary incontinence, poor nutrition, and muscular atrophy are also contributing to risk factors of pressure ulcers. ${ }^{40}$ Some soft tissue or skin infections may also infect SCI patient, including seborrheic dermatitis and fungal infections. ${ }^{41}$

The management can be done by relieving the pressure and doing wound debridement. Antibiotics can be given if wound infection or bacteremia is found. ${ }^{39}$ To prevent this complication, frequently changing position is important followed by using the pressure-relieving device, and regular observation of the skin which is on the bony prominence area. ${ }^{38,39}$ Tissue oxygenation is also an important factor regarding it's important to boost the pressure resistance of the patients. Hand hygiene, contact precautions, and cultural changes are important activities that decreasing the significant number of bacterial infections, notably MRSA and other infections. ${ }^{38}$

$\underline{\text { Pneumonia }}$

Several pulmonary complications can occur throughout SCI, including atelectasis, pneumonia, chest injury, and other respiratory failures or an infection are accountable in causing morbidity and mortality. ${ }^{42}$ Respiratory complications involved in SCI are complex. It includes lung inflammation, vascular impairment, and even still developing an infection. The progress of pneumonia itself may develop shortly after or even in the rehabilitation stage which most of pneumonia occurs. ${ }^{43,44}$ One of the major reasons that pneumonia can occur is because of the inability in doing effective cough. ${ }^{10,42}$ Additional phrenic and intercostal nerve damage may also contribute. The postintubation patient that is attached to a ventilator has a great risk of developing ventilator-associated pneumonia (VAP) and prolong the hospital stay. Antibiotic treatment based on the endotracheal culture is important to choose empiric treatment. ${ }^{10,12,42}$

Lung volume scaled down in the SCI patients with higher levels of inflammation markers IL-6 and C-reactive protein (CRP). ${ }^{45}$ Production of proinflammatory mediators and pulmonary infiltration 
can be reduced by the administration of pharmaceuticals. Furthermore, it may also diminish systemic inflammation and debilitate the risk of pulmonary dysfunction. Administering anti-inflammatory is proven to be critical as it may decrease inflammation, thus, it also accelerates the recovery of pulmonary function in SCI patients. ${ }^{46}$

Retained secretion because of ineffective cough is the most important risk factor. Some methods can be used to handle and prevent this inability, including pulmonary toilet, proper positioning, and cough assistant (frequent suctioning). Extra awareness in oral care is important with the addition of frequent cleaning of the ventilator to diminish the risk of VAP. ${ }^{42,47}$

\section{Bloodstream and other infections}

Bloodstream infection is secondary to other infections, usually urinary, pneumonia, and pressure ulcer. $^{9}$ Several factors decide the outcome of bloodstream infection such as the site of infection, species involved, drug-resistant organisms, and independent factors. Statistically, inadequate therapy in bloodstream infection is not causing any difference in mortality rate. ${ }^{48}$ However, it is still important to administer appropriate antibiotics as this may prolong hospital stay and escalate the costs. ${ }^{14,15,39}$

Osteomyelitis occurrence could happen as the complication of pressure ulcer or spinal surgery. Osteomyelitis can lead to other complications and also raise the cost of the treatments. ${ }^{49}$ Several methods can be chosen to diagnose osteomyelitis, from bone biopsy as the gold standard to MRI which giving high sensitivity and specificity, or even with additional serum investigation (CRP, ESR, PCT). ${ }^{50,51}$ Bone scan also can be used to eliminate osteomyelitis. ${ }^{51}$ Treating osteomyelitis is divided into surgical and non-surgical management. The combination of these two is essential in shortening the duration of the management. ${ }^{49}$ To prevent osteomyelitis, it is important to monitor the source of infection, pressure ulcer should be monitored carefully. If available, hyperbaric oxygen therapy can be used to treat chronic refractory osteomyelitis. ${ }^{49,52}$

Again, immune suppression in post-SCI individuals plays a major role in causing infections. ${ }^{21,27}$ Any usage of blood products, surgical procedures, and injection sites should be taken care of in hygiene. Meningitis may also occur as the result of CSF leakage at the injury scene or post-injury. Acute abdominal emergencies such as gastrointestinal bleeding, peptic ulcer perforation, and pancreatitis are often missed since the loss of sensation circumstances. ${ }^{8}$

\section{Conclusion}

Monitoring post-SCI complications are complex and difficult. Infectious complications have many disadvantages as those could increase morbidity and mortality rates. Immune suppression is hypothesized as one of the main reasons contributing to the incident of infection post-SCI. Not only do infectious complications cause prolonged management but also increases the cost of treatment. Infectious disease as the complication of SCI is important to be noted and should be monitored with a high level of suspicion. Early diagnosis with the addition of appropriate treatment, including non-surgical and surgical interventions, good sanitation and hygiene are important and can be life-saving prevention to decrease morbidity and mortality.

\section{Acknowledgment}

No acknowledgment

\section{References}

1. Mahadewa TGB, Maliawan, S. Clinical aspect and management therapy in spinal cord. Udayana University Press. 2009.

2. Wyndaele M, Wyndaele JJ. Incidence, prevalence and epidemiology of spinal cord injury: what learns a worldwide literature survey? Spinal Cord. 2006;44(9):523-9. DOI: 10.1038/sj.sc.3101893

3. Blair JA, Patzkowski JC, Schoenfeld AJ, et al. Spinal column injuries among Americans in the global war on terrorism. JBJS. 2012;94(18):e135(1-9). DOI: 10.2106/JBJS.K.00502

4. White NH, Black NH. Spinal cord injury (SCI) facts and figures at a glance. Birmingham: National Spinal Cord Injury Statistical Center, Facts and Figures at a Glance. 2016.

5. Kirshblum SC, Burns SP, Biering-Sorensen F, et al. International standards for neurological classification of spinal cord injury (revised 2011). The Journal of Spinal Cord Medicine. 2011;34(6):535-46. DOI: 10.1179/2045772314Y.0000000196

6. Evans CT, LaVela SL, Weaver FM, et al. Epidemiology of hospitalacquired infections in veterans with spinal cord injury and disorder. Infection Control and Hospital Epidemiology. 2008;29(3):234-42. DOI: $10.1086 / 527509$

7. Stanley-Ketting O, Mahadewa T, Maliawan S. Elevation of serum Creactive protein as a predictor for systemic inflammatory response syndrome in cervical spinal cord injury patients. Bali Medical Journal. 2013;2(2):69-71.

8. Abbasi F, Korooni S. Infectious complications after spinal cord injury. Essentials of Spinal Cord Injury Medicine. 2018;13:91. DOI: 10.5772/intechopen.72783 
9. Montgomerie JZ. Infections in patients with spinal cord injuries. Clinical Infectious Diseases. 1997;25(6):1285-90.

10. Lalwani S, Punia P, Mathur P, et al. Hospital acquired infections: preventable cause of mortality in spinal cord injury patients. Journal of Laboratory Physicians. 2014;6(1):36-9. DOI: 10.4103/09742727.129089

11. Hoff JM, Bjerke LW, Gravem PE, et al. Pressure ulcers after spinal cord injury. Tidsskr Nor Laegeforen. 2012;132(7):838-9. DOI: 10.4045/tidsskr.10.0878

12. Sachdeva D, Singh D, Loomba P, et al. Assessment of surgical risk factors in the development of ventilator-associated pneumonia in neurosurgical intensive care unit patients: Alarming observations. Neurology India. 2017;65(4):779-84. DOI: 10.4103/neuroindia.NI_814_16

13. Chacko R, Rajan A, Lionel P, et al. Oral decontamination techniques and ventilator-associated pneumonia. British Journal of Nursing. 2017;26(11):594-9. DOI: 10.12968/bjon.2017.26.11.594

14. Adrie C, Garrouste-Orgeas M, Essaied WI, et al. Attributable mortality of ICU-acquired bloodstream infections: impact of the source, causative micro-organism, resistance profile and antimicrobial therapy. Journal of Infection. 2017;74(2):131-41. DOI: 10.1016/j.jinf.2016.11.001

15. Shindo Y, Hasegawa Y. Regional differences in antibiotic-resistant pathogens in patients with pneumonia: Implications for clinicians. Respirology. 2017;22(8):1536-46. DOI: 10.1111/resp.13135

16. Stein DM, Menaker J, McQuillan K, et al. Risk factors for organ dysfunction and failure in patients with acute traumatic cervical spinal cord injury. Neurocritical Care. 2010;13(1):29-39. DOI: 10.1177/0885066611403270

17. Altinors N. Analysis of serum pro-inflammatory cytokine levels after rat spinal cord ischemia/reperfusion injury and correlation with tissue damage. Turkish Neurosurgery. 2009;19(4):353-9.

18. Anthony DC, Couch Y. The systemic response to CNS injury. Experimental Neurology. 2014;258:105-11.

19. Bao F, Bailey CS, Gurr KR, et al. Increased oxidative activity in human blood neutrophils and monocytes after spinal cord injury. Experimental Neurology. 2009;215(2):308-16. DOI: 10.1016/j.expneurol.2008.10.022

20. Kopp MA, Druschel C, Meisel C, et al. The SCIentinel studyprospective multicenter study to define the spinal cord injury-induced immune depression syndrome (SCI-IDS)-study protocol and interim feasibility data. BMC Neurology. 2013;13(1):168. DOI: 10.1186/14712377-13-168

21. Lucin KM, Sanders VM, Jones TB, et al. Impaired antibody synthesis after spinal cord injury is level dependent and is due to sympathetic nervous system dysregulation. Experimental Neurology. 2007;207(1):75-84
22. Schwab JM, Zhang Y, Kopp MA, et al. The paradox of chronic neuroinflammation, systemic immune suppression, autoimmunity after traumatic chronic spinal cord injury. Experimental Neurology. 2014;258:121-9. DOI: 10.1016/j.expneurol.2007.05.019

23. Popovich P, McTigue D. Damage control in the nervous system: beware the immune system in spinal cord injury. Nature Medicine. 2009;15(7):736-7. DOI: 10.1016/j.expneurol.2014.04.023

24. Brommer B, Engel O, Kopp MA, et al. Spinal cord injury-induced immune deficiency syndrome enhances infection susceptibility dependent on lesion level. Brain. 2016;139(3):692-707. DOI: 10.1093/brain/awv375

25. Riegger T, Conrad S, Schluesener HJ, et al. Immune depression syndrome following human spinal cord injury (SCI): a pilot study. Neuroscience. 2009;158(3):1194-9. DOI: 10.1016/j.neuroscience.2008.08.021

26. Garcia-Arguello LY, O'Horo JC, Farrell A, et al. Infections in the spinal cord-injured population: A systematic review. Spinal Cord. 2017;55(6):526-34. DOI: 10.1038/sc.2016.173

27. Meisel C, Volk HD. Mechanisms of immunodepression after central nervous system injury. In: Mechanisms of Sepsis-Induced Organ Dysfunction and Recovery. Springer: Berlin, Heidelberg. 2007, pp. 335-50.

28. Horst M, Heutschi J, Van Den Brand R, et al. Multisystem neuroprosthetic training improves bladder function after severe spinal cord injury. The Journal of Urology. 2013;189(2):747-53. DOI: 10.1016/j.juro.2012.08.200

29. Cameron AP, Rodriguez GM, Schomer KG. Systematic review of urological followup after spinal cord injury. The Journal of Urology. 2012;187(2):391-7. DOI: 10.1016/j.juro.2011.10.020

30. Tofte N, Nielsen AC, Trøstrup H, et al. Chronic urinary tract infections in patients with spinal cord lesions-biofilm infection with need for long-term antibiotic treatment. Apmis. 2017;125(4):385-91. DOI: 10.1111/apm.12685

31. Karthik L, Kumar G, Keswani T, et al. Protease inhibitors from marine actinobacteria as a potential source for antimalarial compound. PloS One. 2014;9(3):e90972. DOI: 10.1371/journal.pone.0090972

32. Massa LM, Hoffman JM, Cardenas DD. Validity, accuracy, and predictive value of urinary tract infection signs and symptoms in individuals with spinal cord injury on intermittent catheterization. The Journal of Spinal Cord Medicine. 2009;32(5):568-73. DOI: 10.1080/10790268.2009.11754562

33. Moola S, Konno R. A systematic review of the management of shortterm indwelling urethral catheters to prevent urinary tract infections. JBI Database of Systematic Reviews and Implementation Reports. 2010;8(17):695-729. DOI: 10.11124/01938924-201008170-00001

34. Ronco E, Denys P, Bernède-Bauduin C, et al. Diagnostic criteria of urinary tract infection in male patients with spinal cord injury. 
Neurorehabilitation and Neural Repair. 2011;25(4):351-8. DOI: 10.1177/1545968310383432

35. Tenke P, Kovacs B, Johansen TE, et al. European and Asian guidelines on management and prevention of catheter-associated urinary tract infections. International Journal of Antimicrobial Agents. 2008;31:6878. DOI: 10.1016/j.jiantimicag.2007.07.033

36. Nicolle LE. Catheter-related urinary tract infection. Drugs \& Aging. 2005;22(8):627-39. DOI: 10.2165/00002512-200522080-00001

37. Sunn G. Spinal cord injury pressure ulcer treatment: An experiencebased approach. Physical Medicine and Rehabilitation Clinics. 2014;25(3):671-80. DOI: 10.1016/j.pmr.2014.05.002

38. Kruger EA, Pires M, Ngann Y, et al. Comprehensive management of pressure ulcers in spinal cord injury: Current concepts and future trends. The Journal of Spinal Cord Medicine. 2013;36(6):572-85. DOI: 10.1179/2045772313Y.0000000093

39. Pazhouh FK, Parvaz N, Borojeni HR, et al. Prevention of pressure ulcers in spinal cord injury. Journal of Injury and Violence Research. 2012;4(3 Suppl 1):90.

40. Salzberg CA, Byrne DW, Cayten CG, et al. A new pressure ulcer risk assessment scale for individuals with spinal cord injury. American Journal of Physical Medicine \& Rehabilitation. 1996;75(2):96-104. DOI: 10.1097/00002060-199603000-00004

41. Han ZA, Choi JY, Ko YJ. Dermatological problems following spinal cord injury in Korean patients. The Journal of Spinal Cord Medicine. 2015;38(1):63-7. DOI: 10.1179/2045772313Y.0000000154

42. Tollefsen E, Fondenes O. Respiratory complications associated with spinal cord injury. Tidsskr Nor Laegeforen. 2012;132(9):1111-4. DOI: 10.4045/tidsskr.10.0922

43. Veeravagu A, Jiang B, Rincon F, et al. Acute respiratory distress syndrome and acute lung injury in patients with vertebral column fracture (s) and spinal cord injury: A nationwide inpatient sample study. Spinal Cord. 2013;51(6):461-5. DOI: 10.1038/sc.2013.16

44. Yong T, Lili Y, Wen Y, et al. Pulmonary edema and hemorrhage, possible causes of pulmonary infection and respiratory failure in the early stage of lower spinal cord injury. Medical Hypotheses. 2012;79(3):299-301. DOI: 10.1016/j.mehy.2012.05.013

45. Garshick E, Stolzmann KL, Gagnon DR, et al. Systemic inflammation and reduced pulmonary function in chronic spinal cord injury. PMR. 2011;3(5):433-9. DOI: 10.1016/j.pmrj.2011.02.003

46. Bao F, Brown A, Dekaban GA, et al. CD11d integrin blockade reduces the systemic inflammatory response syndrome after spinal cord injury. Experimental Neurology. 2011;231(2):272-83. DOI: 10.1016/j.expneurol.2011.07.001

47. Guo L, Li G, Wang J, et al. Suspicious outbreak of ventilator-associated pneumonia caused by Burkholderia cepacia in a surgical intensive care unit. American Journal of Infection Control. 2017;45(6):660-6. DOI: 10.1016/j.ajic.2017.01.024

48. Saliba M, Saadeh D, Bouchand F, et al. Outcome of bloodstream infections among spinal cord injury patients and impact of multidrugresistant organisms. Spinal Cord. 2017;55(2):148-54. DOI: 10.1038/sc.2016.176

49. Andrianasolo J, Ferry T, Boucher F, et al. Pressure ulcer-related pelvic osteomyelitis: evaluation of a two-stage surgical strategy (debridement, negative pressure therapy and flap coverage) with prolonged antimicrobial therapy. BMC Infectious Diseases. 2018;18(1):166. DOI: 10.1186/s12879-018-3076-y

50. Nolan J, Meregawa PF. The role of serum procalcitonin in establishing diagnosis of bone and joint infections. Journal of Clinical Orthopaedics. 2020;5(1):16-21. DOI: 10.13107/jcorth.2020.v05i01.302

51. Brunel AS, Lamy B, Cyteval C, et al. Diagnosing pelvic osteomyelitis beneath pressure ulcers in spinal cord injured patients: A prospective study. Clinical Microbiology and Infection. 2016;22(3):267-e1. DOI: 10.1016/j.cmi.2015.11.005

52. Goldman RJ. Hyperbaric oxygen therapy for wound healing and limb salvage: A systematic review. PMR. 2009;1(5):471-89. DOI: 10.1016/j.pmrj.2009.03.012 